\title{
USO DE FITOTERÁPICOS EM PACIENTES COM CÂNCER NA REGIÃO DO GRANDE ABC
}

\author{
USE OF PHYTOTHERAPY IN CANCER PATIENTS
}

\begin{abstract}
Camila Ribeiro de Arruda Monteiro ${ }^{1}$, Jean Henri Maselli Schoueri ${ }^{1}$, Karine Corcione Turke ${ }^{1 *}$ (D) , Nicolas Augusto Cabral Ribeiro ${ }^{1}$, Igor Luiz Argani ${ }^{1}$, In Young Noh ${ }^{1}$, Guilherme Querido de Queiroz Ferreira Borrelli ${ }^{1}$, Elaine Shizue Novalo-Goto ${ }^{1}$, Giovanna Cardella Vizentini ${ }^{1}$, João Vitor Maroneze Porfirio ${ }^{1}$, Claudia Vaz de Melo Sette ${ }^{1}$, Daniel de Iracema Gomes Cubero ${ }^{1}$, Auro del Giglio ${ }^{1}$
\end{abstract}

Como citar: Monteiro CRA, Schoueri JHM, Turke KC, et al. USO DE FITOTERÁPICOS EM PACIENTES COM CÂNCER NA REGIÃO DO GRANDE ABC. Clin Onc Let. 2020; Ahead of Print. https://doi.org/10.4322/col.2019.001

\begin{abstract}
RESUMO
Objetivo: Analisar a prevalência do uso dos principais fitoterápicos pela uma população oncológica brasileira atendida pelo Sistema Único de Saúde (SUS), bem como suas indicações e avaliar se houve ou não comunicação à equipe médica do uso dessas substâncias. Métodos: Estudo transversal realizado no serviço de Oncologia da Faculdade de Medicina do $A B C$ com uma amostragem por conveniência. A pesquisa baseou-se na aplicação de questionários a pacientes oncológicos. Resultados: 160 pacientes foram incluídos, dos quais 77 (48,12\%) referiram fazer uso de fitoterápicos. Ao analisar o uso ou não de fitoterápicos, foi observada frequência significativamente maior de uso de fitoterápicos em pacientes casados ou amasiados $(p=0,001)$, menor escolaridade $(p=0,047)$ e procedência do $A B C(p=0,011)$. Não foi observada diferença estatisticamente significante quanto ao sexo, raça, ocupação, naturalidade e religião. Não houve, também, direcionamento do uso de um determinado fitoterápico para algum tipo de câncer específico. 21 (27,27\%) pacientes revelaram a seus respectivos médicos estar sob uso dessas substâncias, e $33(42,28 \%)$ referiram melhora dos sintomas. Conclusão: 0 consumo de fitoterápicos foi frequente na população oncológica estudada, sendo esse uso subnotificado para os médicos responsáveis pelo paciente. Considerando os riscos de interações medicamentosas entre fitoterápicos e o tratamento oncológico convencional, é necessário que se questione a pacientes diretamente quanto ao uso de fitoterápicos.
\end{abstract}

Palavras-chave: Fitoterapia, Oncologia

\begin{abstract}
Objective: To analyze the prevalence of the use of phytotherapy by a Brazilian cancer population attended by the Unified Health System (SUS), as well as their indications and to evaluate whether or not there was communication to the medical team about the use of these substances. Methods: Cross-sectional study conducted at the Oncology Department of ABC Medical School with a convenience sampling. The research was based on the application of questionnaires to cancer patients. Results: 160 patients were included, of which 77 (48.12\%) reported using herbal medicines. When analyzing the use of herbal medicines, it was observed a significantly higher frequency of herbal medicines use in married or divorced patients $(p=0.001)$, lower education $(p=0.047)$ and patients living in $A B C$ cities $(p=0.011)$. No statistically significant difference was observed regarding gender, race, occupation, place of birth and religion. There was also no direct use of a particular herbal medicine for any specific cancer. 21 (27.27\%) patients reported to their respective physicians that they were using these substances, and $33(42.28 \%)$ reported symptom improvement. Conclusion: The use of herbal medicines was frequent in the studied cancer population, and this use was underreported to the doctors responsible for the patient. Considering the risks of drug interactions between herbal medicines and conventional cancer treatment, it is necessary to ask patients directly about the use of herbal medicines.
\end{abstract}

Keyword: Phytotherapy, Medical concology

${ }^{1}$ Faculdade de Medicina do ABC - Av. Lauro Gomes, 2000 - Vila Sacadura Cabral, Santo André - SP, 09060-870

CAAE: 55675916.6 .0000 .0082

Conflito de interesse: Os autores declararam não haver conflitos de interesse que precisam ser informados. 


\section{INTRODUÇÃO}

A medicina não convencional - descrita como todo o conjunto de práticas utilizadas à margem do tratamento convencional, i.e., em conjunto com a terapia clássica previamente estabelecida - tem sido alvo de crescente atenção nas últimas décadas. ${ }^{1}$ Dentre outras categorias desta prática, destaca-se o uso de fitoterápicos que são medicamentos obtidos através de fontes vegetais por métodos naturais. ${ }^{1}$

Entretanto, essas substâncias não são submetidas a estudos rigorosos e também não foram avaliadas e registradas pelos órgãos competentes (como a Agência Nacional de Vigilância Sanitária - ANVISA), o que gera um questionamento em relação a sua eficácia e segurança. ${ }^{2-4}$ No Brasil, os fitoterápicos são amplamente utilizados, em função de fatores como o seu baixo custo, a condição climática - que favorece a abundância da flora local - e à cultura. $^{2}$

Dentro deste universo de usuários, destacam-se os pacientes oncológicos, que frequentemente buscam na medicina complementar alternativas que alimentem a esperança de obter um tratamento melhor, diminuição de sintomas ou até mesmo a cura para suas enfermidades. ${ }^{5}$ Estima-se que uma grande parcela dos pacientes diagnosticados com câncer faça uso de fitoterápicos, com intuito de amenizar e prevenir sintomas relacionados à doença, ${ }^{6,7}$ ou para tratar as condições associadas ao diagnóstico de câncer, tais como ansiedade e depressão. ${ }^{8}$

Apesar deste panorama, as estimativas da prevalência do uso de fitoterápicos nessa população são inconsistentes, variando de 7 a $64 \%$ dos pacientes com câncer. ${ }^{9}$ Ainda, deve-se levar em consideração que apesar de algumas dessas substâncias naturais terem demonstrado efeitos quimiopreventivos e antineoplásicos, é importante que haja cautela quando estas são consumidas simultaneamente ao tratamento oncológico convencional, devido aos riscos de interações medicamentosas. ${ }^{10} \mathrm{O}$ uso indiscriminado e mal aplicado das plantas medicinais pode trazer consequências negativas, podendo interferir na biotransformação dos fármacos convencionais, diminuindo seu efeito ou, até mesmo, aumentando sua toxicidade. ${ }^{11}$

Além disso, a maior porcentagem dos pacientes faz uso dessa terapia complementar sem informar aos profissionais de saúde envolvidos em seu tratamento. Os motivos são diversos, dentre eles: falta de atenção do médico, receio de que haja uma reação negativa, a ideia de que os médicos não possuem conhecimento a respeito das propriedades de tais substâncias. ${ }^{10}$ Neste cenário, é importante estudar também as razões pelas quais pacientes oncológicos buscassem esta alternativa. ${ }^{12,13}$

Desta forma, o presente estudo tem por objetivo analisar a prevalência do uso dos principais fitoterápicos pela população oncológica, se houve ou não melhora subjetiva com o seu uso, suas referidas indicações, e avaliar se deu ou não comunicação à equipe médica do uso dessas substâncias.

\section{MÉTODOS}

\section{DESENHO DO ESTUDO}

Estudo observacional transversal.

Baseado na aplicação de questionário a uma amostragem por conveniência de pacientes atendidos pelo Serviço de Oncologia vinculados a FMABC - i.e., Hospital Estadual Mário Covas, em Santo André e Hospital de Ensino Padre Anchieta, em São Bernardo do Campo. O presente estudo foi questionário utilizado foi previamente aprovado pelo Comitê de Ética em Pesquisa de nossa instituição.

Após assinarem o Termo de Consentimento Livre e Esclarecido (TCLE), os pacientes foram entrevistados e preencheram uma ficha de identificação com seus dados clínicos e demográficos, bem como um questionário sobre o uso de tratamento complementar.

Foram considerados elegíveis para o estudo pacientes que expressaram desejo de participar da pesquisa, com idade igual ou superior a 18 anos, capazes de ler e compreender português, em tratamento oncológico ou a ele submetidos em até 6 meses antecedentes ao estudo. Foram excluídos pacientes que demonstrem não entender a natureza e os procedimentos da pesquisa. 


\section{ANÁLISE ESTATÍ́STICA}

Por se tratar de um trabalho descritivo, após a coleta, os dados foram tabulados e as prevalências analisadas. Para associações entre variáveis qualitativas utilizamos o teste de Qui-quadrado. Todos os cálculos foram realizados com o auxílio do programa Stata ${ }^{\circledR} 12.0$.

\section{RESULTADOS}

No período de maio de 2016 a agosto de 2017 foram incluídos um total de 160 pacientes, do quais 95 (59,38\%) eram do sexo feminino e 65 (40,62\%) do sexo masculino. Do total, 77 (48,12\%) referiram fazer uso de fitoterápicos. As características sociodemográficas dos pacientes incluídos seguem na tabela 1.

Tabela 1 - Dados Sociodemográficos dos Pacientes que usam e não usam fitoterápicos

\begin{tabular}{|c|c|c|c|c|c|}
\hline & & $\begin{array}{l}\text { Paciente } \\
\text { (Total) }\end{array}$ & $\begin{array}{c}\text { Faz uso de } \\
\text { Fitoterápicos }\end{array}$ & $\begin{array}{c}\text { Não faz uso } \\
\text { de } \\
\text { Fitoterápicos }\end{array}$ & $\mathbf{P}$ \\
\hline \multirow{3}{*}{ Sexo } & Feminino & 95 & 49 & 46 & \multirow{2}{*}{0,290} \\
\hline & Masculino & 65 & 28 & 37 & \\
\hline & Brancos & 70 & 36 & 34 & \multirow{3}{*}{0,529} \\
\hline \multirow[t]{3}{*}{ Raça } & Pardos & 68 & 33 & 35 & \\
\hline & Negros & 19 & 7 & 12 & \\
\hline & Amaziado/Casado & 43 & 10 & 33 & \multirow{3}{*}{0,001} \\
\hline \multirow[t]{3}{*}{ Estado Civil } & Solteiro/Divorciado & 71 & 41 & 30 & \\
\hline & Viúvo & 46 & 26 & 20 & \\
\hline & $\begin{array}{l}\text { Ensino Fundamental } \\
\text { Completo/Incompleto }\end{array}$ & 105 & 56 & 49 & \multirow{3}{*}{0,047} \\
\hline \multirow[t]{2}{*}{ Escolaridade } & $\begin{array}{c}\text { Ensino Médio } \\
\text { Completo/Incompleto }\end{array}$ & 46 & 15 & 31 & \\
\hline & $\begin{array}{c}\text { Ensino Superior } \\
\text { Completo/Incompleto }\end{array}$ & 9 & 6 & 3 & \\
\hline \multirow{2}{*}{ Ocupação } & Trabalha & 69 & 28 & 41 & \multirow{2}{*}{0,096} \\
\hline & Não Trabalha & 91 & 49 & 42 & \\
\hline \multirow{2}{*}{ Naturalidade } & São Paulo & 77 & 38 & 39 & \multirow{2}{*}{0,765} \\
\hline & Outros & 83 & 39 & 44 & \\
\hline \multirow[t]{4}{*}{ Procedência } & $A B C$ & 133 & 70 & 63 & \multirow{4}{*}{0,011} \\
\hline & Outros & 27 & 7 & 20 & \\
\hline & Católica & 90 & 41 & 49 & \\
\hline & Evangélica & 9 & 6 & 3 & \\
\hline \multirow[t]{6}{*}{ Religião } & Espírita & 44 & 21 & 23 & \multirow[t]{6}{*}{0,687} \\
\hline & Outras & 2 & 1 & 1 & \\
\hline & Sem Religião & 4 & 1 & 3 & \\
\hline & Mama & 48 & 18 & 30 & \\
\hline & Trato gastrointestinal & 37 & 11 & 26 & \\
\hline & Trato genitourinário & 27 & 6 & 21 & \\
\hline \multirow[t]{4}{*}{ Tipo de câncer } & Neoplasias hematológicas & 23 & 6 & 17 & \multirow[t]{4}{*}{0.406} \\
\hline & Ginecológico & 9 & 2 & 7 & \\
\hline & Pulmão & 8 & 1 & 7 & \\
\hline & Outros & 8 & 2 & 6 & \\
\hline
\end{tabular}


Ao analisar o uso ou não de fitoterápicos, foi observada frequência significativamente maior de uso de fitoterápicos em pacientes casados ou amasiados $(p=0,001)$, menor escolaridade $(p=0,047)$, procedência do $A B C$ $(p=0,011)$. Não foi observada diferença estatisticamente significante quanto ao sexo, raça, ocupação, naturalidade e religião ou tipo de câncer.

A tabela 2 relaciona todos os fitoterápicos citados pelos pacientes, e os referidos motivos e/ou supostos efeitos pelos quais empregaram tais substâncias.

Tabela 2: Fitoterápicos utilizados pelos pacientes relacionados aos respectivos motivos e/ou efeitos referidos pelos quais foram utilizados.

\begin{tabular}{|c|c|}
\hline FITOTERÁPICO & EFEITO REFERIDO \\
\hline CAMOMILA & ANTI-INFLAMATORIO, QUEIMADURA (RT), CALMANTE \\
\hline GUAÇATONGA & DIURÉTICO \\
\hline UNHA DE GATO & ATENUA PROCESSO ALÉRGICO VIA AÉREA \\
\hline CHÁ DE CAVALINHO & GASTRITE \\
\hline PRÓPOLIS VERDE & COMBATE CA \\
\hline ESPINHEIRO SANTO & NAÚSEA \\
\hline ARNICA & ANTI-INFLAMATÓRIO; DOR; CALMANTE \\
\hline YOMOGUI & COMBATE CA \\
\hline CAPIM SANTO & CALMANTE; DIURÉTICO, CÓLICA \\
\hline GRAVIOLA & $\begin{array}{c}\text { MELHORA TOSSE; DISPNÉIA; AUXILIA NO TRATAMENTO } \\
\text { ONCOLÓGICO; MELHORA SISTEMA IMUNE; COMBATE O } \\
\text { CÂNCER }\end{array}$ \\
\hline NONE & COMBATE O CÂNCER \\
\hline AROEIRA & CICATRIZAÇÃO (CIRÚRGICA) \\
\hline AÇAI; PINHA & MELHORA SISTEMA IMUNE; ENERGÉTICO \\
\hline FOLHA MARACUJA E AMORA & CALMANTE \\
\hline FLORAL & CALMANTE; MELHORA SISTEMA IMUNE \\
\hline SUCUPIRA & OSTEOPOROSE \\
\hline GINCOBILOBA & LABIRINTITE \\
\hline FLORAL & MELHORA SIT IMUNE; CALMANTE \\
\hline AÇAFRÃO & COMBATE O CÂNCER \\
\hline BABOSA & COMBATE O CÂNCER \\
\hline AVOEIRA & CICATRIZANTE; AJUDA NA INFECÇÃO \\
\hline
\end{tabular}

Por fim, é digno de nota que 33 pacientes $(42,28 \%)$ afirmaram sentir melhora dos sintomas ao fazerem uso de fitoterápicos, e apenas $21(27,27 \%)$ revelaram a seus respectivos médicos estar sob uso dessas substâncias.

\section{DISCUSSÃO}

Estudos na literatura divergem quanto à frequência do uso de fitoterápicos por pacientes oncológicos. Uma revisão da literatura incluindo estudos realizados no Reino Unido identificou que o uso de medicina alternativa variava de 3,1 a $24,9 \%$ dentre os pacientes oncológicos. ${ }^{14}$ Por outro lado, um estudo realizado na Nigéria observou que até $65 \%$ dos pacientes faziam uso desse tipo de medicação. ${ }^{15} \mathrm{Em}$ outra revisão da literatura, foi encontrado uma prevalência variando de 7 a $64 \% .{ }^{9}$ No presente estudo, $48,12 \%$ dos pacientes inclusos afirmaram fazer o uso de ao menos um tipo de fitoterápico, o que se situa em posição intermediária aos extremos de prevalência do seu uso descritos na literatura.

Observou-se também uma diferença estatística significante em relação ao grau de escolaridade entre os usuários e não usuários dessa terapia. Em nosso estudo, pacientes com menor grau de escolaridade tiveram maior prevalência do uso de fitoterápicos. Este achado poderia ser explicado por um nível sócio econômico menor de 
pacientes com nível educacional menor e/ou pelo seu relativo baixo custo em nosso meio. Nossos dados divergem do que já foi observado anteriormente por outros autores que descreveram um maior uso de fitoterápicos entre pacientes de maior nível sócio econômico. ${ }^{16,17}$ Ainda, dentre os pacientes que fazem uso dessa terapia complementar, 63,63\% são do sexo feminino, característica que se assemelha ao relatado em trabalhos prévios. ${ }^{9,16,17}$

Como demonstrado nos resultados, não pareceu haver um uso direcionado de um fitoterápico especifico para um determinado tipo de câncer. Pelo contrário, observou-se variados motivos relacionados ao uso dessas substâncias, tais como a redução de sintomas relacionados ao câncer ou a seu tratamento. Curiosamente, dentre os estudos relacionados ao uso de medicina alternativa por pacientes oncológicos, Gratus $\mathrm{C}$ et all ${ }^{14}$ destacam que geralmente não se leva em consideração as características e motivações do uso de cada tipo de substância. Dessa forma, o presente estudo visou destacar tais informações, identificando que os motivos são variáveis, e muitas vezes não relacionados a um efeito conhecido da substância utilizada.

Considerando as preocupações no uso de medicina alternativa, ressalta-se a possibilidade de interação medicamentosa dos fitoterápicos com tratamentos médicos convencionais. ${ }^{18}$ Apesar disso, muitas dessas interações não são bem documentadas devido à falta de trabalhos voltados a esse tema. ${ }^{19} \mathrm{Em}$ um estudo com 5052 participantes de Elmer GW el at, ${ }^{18}$ identificou-se que o uso de medicina alternativa era comum, sendo que até $5,8 \%$ dos pacientes apresentavam alto risco de interação adversa, principalmente sangramento. Especificamente no meio oncológico, são conhecidas graves interações relacionadas a fitoterápicos e quimioterapia, ${ }^{19}$ de modo que o médico deve estar atento ao uso dessas substâncias por seus pacientes. O risco de efeitos adversos torna-se ainda maior considerando que muitos pacientes não relatam a seus médicos sobre o uso deste tipo de medicina alternativa. No presente estudo, a minoria dos pacientes que faziam uso de fitoterápicos avisaram aos médicos acerca de seu o que já foi reportado por outros estudos envolvendo pacientes oncológicos. ${ }^{20}$ Dessa forma, é fundamental a busca ativa dos médicos quanto ao uso de fitoterápicos por seus pacientes para prevenir interações medicamentosas indesejáveis.

Cabe ressaltar que a maioria dos pacientes incluídos no estudo referiu melhora dos sintomas apresentados ao fazer uso de fitoterápicos. Assim, além de se considerar o possível efeito placebo, não podemos excluir que mesmo que por mecanismos ainda pouco compreendidos, tais substâncias possam apresentar efeitos benéficos. Acreditamos que fitoterápicos devem ser melhor estudados no contexto de estudos controlados no futuro com vista a melhora da qualidade de vida, especialmente em pacientes com neoplasias malignas. ${ }^{21-23}$

Dentre as limitações do presente estudo, destaca-se a possibilidade de viés de memória, visto que um estudo de prevalência depende do uso relatado pelo paciente e o fato de nossa amostragem ter sido por conveniência e não consecutiva

\section{CONCLUSÃO}

O consumo de fitoterápicos foi frequente na população oncológica, sendo esse uso subnotificado para os médicos responsáveis pelo paciente. Médicos devem ser cientificados por seus pacientes acerca do uso de fitoterápicos pelo risco de interações medicamentosas.

\section{REFERÊNCIAS}

1. Elias MC, Alves E. Medicina não-convencional: prevalência em pacientes oncológicos. Rev Bras Cancerol. 2002;48(4):523-32.

2. Fukumasu H, Latorre AO, Bracci N, Górniak SL, Dagli MLZ. Fitoterápicos e potenciais interações medicamentosas na terapia do câncer. Revista Brasileira de Toxicologia. 2008;21(2):49-59.

3. Brasil. Agência Nacional de Vigilância Sanitária. Resolução de Diretoria Colegiada (RDC) no 48, de 16 de março de 2004. Dispõe sobre o registro de medicamentos fitoterápicos. Diário Oficial da União [Internet]; Brasília; 18 mar 2012 [citado 2015 nov 8]. Disponível em: http://www.anvisa.gov.br/medicamentos/fitoterapicos/orientacao_RDC48.pdf

4. Carvalho ACB, Balbino EE, Maciel A, Perfeito JPS. Situação do registro de medicamentos fitoterápicos no Brasil. Rev Bras Farmacogn. 2008;18(2):314-9. http://dx.doi.org/10.1590/S0102-695X2008000200028. 
5. Damery S, Gratus C, Grieve R, et al. The use of herbal medicines by people with cancer: a cross-sectional survey. Br J Cancer. 2011;104(6):927-33. http://dx.doi.org/10.1038/bjc.2011.47. PMid:21364591.

6. Ali N, Hussain-Gambles M. Complementary and alternative medicine (CAM) use among South Asian patients with cancer in Britain. Diversity Health Social Care. 2005;2(1):41-5.

7. Vickers KA, Jolly KB, Greenfield SM. Herbal medicine: women's views, knowledge and interaction with doctors: a qualitative study. BMC Complement Altern Med. 2006;6(1):40. http://dx.doi.org/10.1186/1472-6882-6-40.

PMid:17156416.

8. Verhoef MJ, Hilsden RJ, O’Beirne M. Complementary therapies and cancer care: an overview. Patient Educ Couns. 1999;38(2):93-100. http://dx.doi.org/10.1016/S0738-3991(99)00056-7. PMid:14528701.

9. Ernst $E$, Cassileth BR. The prevalence of complementary/alternative medicine in cancer: a systematic review. Cancer. 1998;83(4):777-82. http://dx.doi.org/10.1002/(SICI)1097-0142(19980815)83:4<777::AID-CNCR22>3.0.CO;2-O. PMid:9708945.

10. Meijerman I, Beijnen JH, Schellens JH. Herb-drug interactions in oncology: focus on mechanisms of induction. Oncologist. 2006;11(7):742-52. http://dx.doi.org/10.1634/theoncologist.11-7-742. PMid:16880233.

11. Johns Hopkins Medical Institutions. The role of alternative treatments for cancer. Johns Hopkins Med Lett Health After 50. 2000;11(12):6-7. PMid:10732503.

12. Burstein HJ. Discussing complementary therapies with cancer patients: what should we be talking about? J Clin Oncol. 2000;18(13):2501-4. http://dx.doi.org/10.1200/JCO.2000.18.13.2501. PMid:10893279.

13. Leal F, Schwartsmann G, Lucas HS. Medicina complementar e alternativa: uma prática comum entre os pacientes com câncer. Rev Assoc. Bras. 2008;54(6):481-2. http://dx.doi.org/10.1590/S0104-42302008000600007.

14. Gratus C, Damery S, Wilson S, et al. The use of herbal medicines by people with cancer in the UK: a systematic review of the literature. Q J Med. 2009;102(12):831-42. http://dx.doi.org/10.1093/qjmed/hcp137. PMid:19797394.

15. Schwartsmann G. Developing anticancer agents from natural sources. Clin Adv Hematol Oncol. 2006;5(5):359-61. PMid:16830424.

16. Harris P, Finlay IG, Cook A, Thomas KJ, Hood K. Complementary and alternative medicine use by patients with cancer in Wales: a cross-sectional survey. Complement Ther Med. 2003;11(4):249-53. http://dx.doi.org/10.1016/S0965-

2299(03)00126-2. PMid:15022659.

17. Chao MT, Wade CM. Socioeconomic Factors and Women's Use of Complementary and Alternative Medicine in Four Racial/Ethnic Groups. Ethn Dis. 2008;18(1):65-71. PMid:18447102.

18. Elmer GW, Lafferty WE, Tyree PT, Lind BK. Potential Interactions between complementary/alternative products and conventional medicines in a medicare population. Ann Pharmacother. 2007;41(10):1617-24.

http://dx.doi.org/10.1345/aph.1K221. PMid:17785609.

19. Yap KY, See CS, Chan A. Clinically-relevant chemotherapy interactions with complementary and alternative medicines in patients with cancer. Recent Pat Food Nutr Agric. 2010;2(1):12-55. http://dx.doi.org/10.2174/2212798411002010012. PMid:20653549.

20. Ge J, Fishman J, Vapiwala N, et al. Patient-physician communication about complementary and alternative medicine in a radiation oncology setting. Int J Radiat Oncol Biol Phys. 2013;85(1):e1-6. http://dx.doi.org/10.1016/j.ijrobp.2012.08.018. PMid:23040545.

21. Yin S-Y, Wei W-C, Jian F-Y, Yang N-S. Therapeutic applications of herbal medicines for cancer patients. Evid Based Complement Alternat Med. 2013;2013:302426. http://dx.doi.org/10.1155/2013/302426. 
22. Gerber B, Scholz C, Reimer T, Briese V, Janni W. Complementary and alternative therapeutic approaches in patients with early breast cancer: a systematic review. Breast Cancer Res Treat. 2006;95(3):199-209.

http://dx.doi.org/10.1007/s10549-005-9005-y. PMid:16254687.

23. Harlan WR Jr. Research on complementary and alternative medicine using randomized controlled trials. J Altern Complement Med. 2001;7(Suppl 1):45-52. http://dx.doi.org/10.1089/107555301753393805. PMid:11822635.

* Autora correspondente:

Karine Corcione Turke

Faculdade de Medicina do ABC. Avenida Lauro Gomes, 2000, Vila Príncipe de Gales, Santo André - SP, Brasil. CEP: 09060-870

Telefone: +55 11 97393-8012.

E-mail: karineturke@hotmail.com 